\title{
Troponina en el diagnóstico de infarto al miocardio: Consideraciones desde el laboratorio clínico
}

\author{
ANA MARÍA GUZMÁN D., TERESA QUIROGA G.
}

\section{Troponin in the diagnosis of myocardial infarction: An approach from the Clinical Laboratory}

Among the many biomarkers studied to evaluate myocardial damage, troponin is considered the most sensitive and specific. However, current methodologies present pre-analytical, analytical and post-analytical problems, of which the more significant are the lack of standardization and a high uncertainty in the level of decision or cutoff. It is hoped that a new generation of assays called "ultrasensitive" will improve analytical performance characteristics of the technique and achieve compliance with the internationally recommended quality specifications.

(Rev Med Chile 2010; 138: 379-382).

Key words: Biological markers; Myocardial infarction; Troponin.

\section{RESUMEN}

Entre los múltiples biomarcadores estudiados para evaluar daño miocárdico, la troponina se considera el más sensible y específico. Sin embargo, la mayoría de las metodologías actuales utilizadas para su determinación presentan problemas tanto pre-analíticos, analíticos como post-analíticos, de los cuales los más relevantes son la falta de estandarización y una imprecisión alta en el nivel de decisión o de corte. Se espera que una nueva generación de ensayos para la determinación de troponina, denominados ultrasensibles, mejoren las características de desempeño de la técnica y logren cumplir con las especificaciones de calidad recomendadas internacionalmente.

\section{DE LA VIDA REAL}

Un paciente consulta en el Servicio de Urgencia por un cuadro de dolor anginoso de 6 horas de evolución, sin cambios electrocardiográficos. Se le solicita troponina en sangre la cual resulta de 0,05 $\mathrm{ng} / \mathrm{mL}$ (limite de referencia hasta $0,04 \mathrm{ng} / \mathrm{mL}$ ). Ingresa para estudio con sospecha de un infarto al miocardio en evolución. La determinación se repite 4 y 8 horas después mostrando niveles de troponina de 0,03 ng/mL y 0,02 ng/mL, manteniéndose el paciente sin cambios en el electrocardiograma. ¿Cómo se podría explicar esta diferencia de valores en la determinación de troponina? 
Troponina en infarto al miocardio: Consideraciones desde el laboratorio clínico - A.M. Guzmán D. et al

L as enfermedades cardiovasculares son en la actualidad una de las principales causas de morbi-mortalidad, especialmente en países desarrollados. Según las estadísticas entregadas por el Ministerio de Salud correspondientes al año 2006, la primera causa de muerte en Chile son las enfermedades isquémicas del corazón, con una tasa de 48,3/100.000 habitantes ${ }^{1}$. En los últimos años se han incorporado múltiples marcadores de enfermedad cardiovascular para su uso clínico, tanto para evaluar daño miocárdico, función miocárdica y factores de riesgo cardiovascular ${ }^{2}$.

En el año 2001 los National Institutes of Health $(\mathrm{NIH})$ establecieron como definición de biomarcador "cualquier indicador de un proceso normal o patológico, o de la respuesta farmacológica a una intervención terapéutica que pueda ser objetivamente medido y evaluado" ". Un biomarcador puede ser medido en una muestra de sangre, orina, etc., pero también puede ser un registro obtenido de un paciente, como la medición de presión arterial, un registro electrocardiográfico, etc.

Un biomarcador ideal debe tener ciertas características (Tabla 1) cuya relevancia va a depender de su aplicación. Si el biomarcador va a ser utilizado como examen de tamizaje o screening, la técnica debe ser altamente sensible, con especificidad aceptable, de bajo costo, fácil de realizar y con un alto valor predictivo negativo. $\mathrm{Si}$, en cambio, el biomarcador va a ser utilizado para el monitoreo de un paciente respecto a la progresión de una enfermedad o la respuesta a una terapia, es importante que el analito tenga una baja variabilidad biológica, ya que el valor inicial del paciente pasa a ser su referencia o control ${ }^{4}$.

\section{Tabla 1. Características de un biomarcador ideal}

1. Aceptabilidad por parte del paciente
2. Estabilidad "in vivo" e "in vitro"
3. Sensibilidad analítica y funcional adecuada
4. Reproducibilidad y exactitud adecuada
5. Laboriosidad mínima, idealmente automatizado
6. Estandarización internacional
7. Baja variación biológica
8. Valores de referencia por género, sexo y etnia
9. Buena exactitud diagnóstica y valor pronóstico
10. Buena relación costo/beneficio

\section{Troponina}

La troponina es una proteína globular de gran tamaño (aprox. 70.000 daltons) reguladora de la contracción del músculo cardíaco. Contiene tres subunidades polipeptídicas: troponina C (fijadora de calcio), troponina I (inhibidora de la interacción actina-miosina) y troponina $\mathrm{T}$ (fijadora de tropomiosina), las cuales son liberadas hacia el torrente sanguíneo durante un infarto al miocardio, por pérdida de la integridad de la membrana celular.

La introducción en el año 1989 de un inmunoensayo para su medición dio lugar a la detección de lesiones microscópicas e incluso a una nueva definición de infarto ${ }^{5}$, por lo que su medición está fuertemente recomendada en la literatura internacional. Actualmente existen inmunoensayos para la detección tanto de troponina I como $\mathrm{T}$, mostrando ambas moléculas características diagnósticas similares, considerándose los biomarcadores más sensibles y específicos de daño miocárdico. Los datos acumulados indican que tanto troponina I como $\mathrm{T}$ aparecen en el suero entre 4 y 10 horas después del inicio del infarto, tienen su peak entre las 12 y $48 \mathrm{~h}$, permaneciendo elevadas entre 4 y 10 días.

A pesar de su amplia utilización en clínica, existe preocupación por problemas tanto de tipo pre-analítico como analítico y post-analítico ${ }^{6}$.

\section{Problemas de la fase pre-analítica}

A pesar de que los métodos disponibles comercialmente para la medición de troponina aceptan como matriz del ensayo tanto suero como plasma, se han encontrado diferencias significativas, siendo los valores hasta $30 \%$ menores en el plasma comparado con el suero. El EDTA puede romper complejos de troponina $\mathrm{Ca}++$ dependientes y disminuir las concentraciones de troponina en ensayos que miden estas formas moleculares. Se recomienda, entonces, que el tipo de muestra debe ser consistente, al menos para el seguimiento de un determinado paciente ${ }^{7}$.

\section{Problemas de la fase analítica}

Estandarización: En marcadores tan relevantes como la troponina, es importante que éstos sean medidos con métodos estandarizados, comparables o armonizados. Sin embargo, esto aún no se cumple, por lo que es posible encontrar metodo- 
logías que difieren en sus valores de corte o cutoff y en sus valores de sensibilidad analítica en más de dos órdenes de magnitud.

Esto puede explicarse por el uso de estándares de calibración diferentes, lo cual debe corregirse logrando que los fabricantes utilicen un calibrador común (calibrador secundario) e introduciendo el uso de un suero conmutable (que se comporte como muestra clínica con un valor asignado conocido) con el cual realizar las comparaciones inter-laboratorio necesarias. Sin embargo, las diferencias antes mencionadas también pueden ser explicadas por diferencias en el diseño del anticuerpo monoclonal utilizado. La molécula de troponina es sensible a proteólisis en su extremo $\mathrm{N}$-(amino) y C-(carboxi) terminal siendo el fragmento medio, entre los aminoácidos 30 al 110 , más estable. La proteólisis progresiva induce la circulación de una mezcla compleja de formas moleculares, incluyendo formas binarias o ternarias y también libres. Idealmente, los ensayos deben reconocer las diferentes formas, acomplejadas y libres, de igual manera. Sin embargo, se ha demostrado una inmunoreactividad variable a las distintas isoformas, lo cual podría resultar en una sobre- o sub-estimación de la verdadera concentración de troponina en la muestra. La International Federation of Clinical Chemistry y su Comité de Estandarización de Marcadores Cardíacos ha recomendado que los proveedores utilicen anticuerpos dirigidos a epítopes ubicados en el fragmento medio de la molécula ${ }^{7}$.

Limite de detección e imprecisión analítica: Los valores de imprecisión intra-corrida y total (reproducibilidad) son también muy variables, dependiendo del método utilizado. La recomendación actual es que el ensayo utilizado tenga un nivel de imprecisión $\leq$ a $10 \%$ en el nivel de decisión (cutoff o punto de corte) y que el límite de detección sea al menos 5 veces menor al nivel de decisión utilizado.

En un estudio realizado en el año 2006 con 16 ensayos diferentes, sólo tres cumplían esta condición ${ }^{6}$. Una nueva generación de ensayos para troponina denominados ultrasensibles, que pueden medir concentraciones un orden de magnitud menor a los actualmente disponibles (de microgramos a picogramos) se han desarrollado y se están introduciendo lentamente para mejorar las características de desempeño analítico, de acuerdo a las guías internacionales y especificaciones de calidad $^{8,9}$.

Limite de referencia: Un valor anormal de un biomarcador puede ser establecido a través de tres aproximaciones diferentes: a) obtener un valor de discriminación que separe pacientes sanos de enfermos; b) obtener un valor "umbral" que identifique un cierto nivel de riesgo para desarrollar una enfermedad y c) obtener un límite de referencia establecido por un análisis estadístico de la distribución de los valores del biomarcador en una población sana dividida en percentiles, fijando el valor de referencia en un determinado percentil, clásicamente $95,97,5$ ó 99\%. El percentil utilizado como valor de referencia, se selecciona en base a las implicancias médicas, éticas, económicas, etc. que tenga el biomarcador en cuestión, asumiendo en cada percentil un porcentaje de falsos positivos o negativos diferente. De acuerdo a las guías internacionales, para troponina se recomienda trabajar con el percentil 99\% de la población de referencia ${ }^{6,7}$.

\section{Problemas de la fase post-analítica}

Los actuales criterios para el diagnóstico de infarto consideran, además de la evidencia clínica de isquemia, el incremento o disminución de la concentración de troponina, con al menos un valor por sobre el percentil 99\% de la población de referencia. La evaluación seriada de niveles de troponina podría ser útil en pacientes sin cambios electrocardiográficos o en casos de dolor precordial atípico.

La interpretación por parte del médico de la concentración de troponina en los niveles de decisión o cutoff debe ser cuidadosa y siempre con conocimiento de la imprecisión del método utilizado localmente.

\section{POCT}

Es importante tener claro que los ensayos en formato POCT (Point of Care Testing o exámenes al lado de la cama del enfermo) para troponina generalmente carecen de la sensibilidad y precisión analítica requeridas en el nivel del percentil 99\%, mostrando un coeficiente de variación mayor al $10 \%$. Aún cuando las plataformas POCT son una importante herramienta por su rápido tiempo de respuesta, se requiere una mejoría sustancial de 
sus características de desempeño, antes de introducirlos a su uso en la práctica clínica.

\section{Criterios de trabajo en el laboratorio}

Desde el punto de vista del laboratorio, se deben establecer procedimientos de control de calidad estrictos para obtener una técnica reproducible, especialmente a niveles de concentración baja. Se requiere la monitorización estrecha de la técnica al nivel de decisión o cutoff. Si el proveedor o fabricante no cuenta con nivel bajo de control, se puede usar un control de tercera parte (de otro fabricante) o, incluso, un pool de suero almacenado congelado y fraccionado en alícuotas. La frecuencia de la calibración debe ser definida en base a la imprecisión del método, usando preferentemente varios puntos de calibración, especialmente a niveles bajos de concentración de troponina.

\section{Cierre del caso}

En el caso planteado de la vida real, el paciente no presentó en su evolución final un infarto del miocardio. El primer valor de troponina de $0,05 \mathrm{ng} / \mathrm{mL}$ se podría haber explicado porque la técnica utilizada en el laboratorio presentaba un coeficiente de variación mayor al $10 \%$ en el nivel de decisión o de corte $(0,04 \mathrm{ng} / \mathrm{mL})$ y que corresponde, como ya se mencionó, a lo recomendado internacionalmente.

\section{Conclusiones}

La introducción de técnicas de medición de troponina con mayor sensibilidad analítica y mejor precisión es una necesidad ampliamente reconocida en la literatura. Para evaluar adecuadamente estas nuevas metodologías se requiere la estrecha colaboración entre el laboratorio y los médicos clínicos, asegurando una adecuada validación y el establecimiento local de los valores de referencia con sujetos sanos desde el punto de vista cardiovascular.

\section{Referencias}

1. Ministerio de Salud, Departamento de Estadísticas e Información de Salud. Mortalidad de ambos sexos, según principales causas específicas de defunción, Chile 2006. Disponible en: http://www.minsal.cl/ [Consultado el 16 de noviembre de 2009].

2. Morrow DA, Cannon CP, Jesse RL, Newby LK, Ravkilde J, Storrow AB, et al. National Academy of Clinical Biochemistry Laboratory Medicine Practice Guidelines: Clinical characteristics and utilization of biochemical markers in acute coronary syndromes. Circulation 2007; 115: 356-75

3. Biomarkers Definition Working Group. Biomarkers and surrogate endpoints: preferred definitions and conceptual framework. Clin Pharmacol Ther 2001; 69: 89-95.

4. Vittorini S, Clerico A. Cardiovascular biomarkers: increasing impact of laboratory medicine in cardiology practice. Clin Chem Lab Med 2008; 46: 748-63.

5. Thygesen K, Alpert JS, White HD; Joint ESC/ACCF/ AHA/WHF Task Force for the Redefinition of Myocardial Infarction. Universal definition of myocardial infarction. J Am Coll Cardiol 2007; 50: 2173-95.

6. Tate JR. Troponin revisited 2008: assay performance. Clin Chem Lab Med 2008; 46: 1489-500.

7. Panteghini M, Gerhardt W, Apple FS, Dati F, Ravkilde J, Wu AH. Quality specifications for cardiac troponin assays. Clin Chem Lab Med 2001; 39: 175-9.

8. Melanson SE, Morrow DA, Jarolim P. Earlier detection of myocardial injury in a preliminary evaluation using a new troponin I assay with improved sensitivity. Am J Clin Pathol 2007; 128: 282-6.

9. Wu AH, Jaffe AS. The clinical need for high-sensitivity cardiac troponin assays for acute coronary syndromes and the role for serial testing. Am Heart J 2008; 155: 208-14. 